\title{
miR-223-3p reduces high glucose and high fat-induced endothelial cell injury in diabetic mice by regulating NLRP3 expression
}

\author{
BO DENG ${ }^{1}$, YING HU ${ }^{1}$, XIA SHENG ${ }^{1}$, HUIJUN ZENG ${ }^{2}$ and YANAN HUO ${ }^{3}$ \\ Departments of ${ }^{1}$ Endocrinology and ${ }^{2}$ Pharmacy, The Third Affiliated Hospital \\ of Nanchang University, Nanchang, Jiangxi 330008; ${ }^{3}$ Department of Endocrinology, \\ Jiangxi Provincial People's Hospital, Nanchang, Jiangxi 330006, P.R. China
}

Received June 12, 2019; Accepted December 18, 2019

DOI: $10.3892 /$ etm.2020.8864

\begin{abstract}
Expression levels of miR-223-3p and NLRP3 in high glucose and high fat (HGHF)-induced diabetic mice, and the mechanism on the injury of mouse cardiac microvascular endothelial cells (MCMECs) were investigated. Four-week C57BL/6J laboratory mice were selected and randomized into a control group and a model group $(\mathrm{n}=10$ each). Mice in the model group were fed with HGHF diet to establish a mouse model of diabetes. Further MCMECs were purchased to construct carriers through transient transfection, and were separated into a normal group (cultured in the normal environment), a model group (not transfected), a blank carrier group (transfected with miR-NC), a miR-223-3p-mimics group, and a miR-223-3p-inhibitor group. RT-qPCR was used to detect the expression levels of miR-223-3p and NLRP3, and western blot analysis to detect the expression levels of NLRP3, apoptosis-related proteins Bax and caspase-3, and anti-apoptotic protein Bcl-2. Flow cytometry was used to observe apoptosis and TargetScan to predict the target relationship between miR-223-3p and NLRP3. Dual-luciferase reporter gene assay was used to detect the relationship between miR-223-3p and NLRP3. Compared with those in the control group, the mice in the model group had significantly lower expression of miR-223-3p. However, significantly higher mRNA and protein expression levels of NLRP3 were observed $(\mathrm{P}<0.05)$. After modeling, miR-223-3p overexpression downregulated the expression levels of NLRP3 mRNA, Bax and NLRP3 protein, as well as inhibited endothelial cell apoptosis $(\mathrm{P}<0.05)$, while the inhibition of miR-223-3p expression upregulated the expression levels and promoted
\end{abstract}

Correspondence to: Dr Yanan Huo, Department of Endocrinology, Jiangxi Provincial People's Hospital, 92 Aiguo Road, Nanchang, Jiangxi 330006, P.R. China

E-mail: hyiv13@163.com; huoyanan_jxyy@sina.com

Key words: miR-223-3p, Nod-like receptor protein 3, endothelial cells apoptosis. In conclusion, miR-223-3p expression is low, however, NLRP3 is highly expressed in the heart tissue of HGHF-induced diabetic mice. miR-223-3p reduces the injury of MCMECs and inhibits endothelial cell apoptosis in mice by regulating the expression of NLRP3.

\section{Introduction}

As social environment and living habits change, the incidence of diabetes has increased (1). Heart disease is a major complication of this condition. Diabetic patients have reduced cardiac function with disease progression, and eventually experience heart failure. Thus, cardiovascular functional disorder is also a major cause of death $(2,3)$. Cardiovascular diseases in diabetic patients begin with vascular endothelium, and hyperglycemia and hyperlipidemia in the body fluids are the leading causes of endothelial cell injury (4). Vascular endothelial cell injury has already occurred in patients with early diabetes, so intervention in the injury is significant for the patients (5).

MicroRNA, a research hotspot, is a non-coding single stranded small molecule RNA and a marker for cell damage. miR-223-3p is also a recent hotspot in cardiovascular diseases $(6,7)$. A study on a mouse model of sepsis revealed that the deletion of miR-223-3p promotes myocardial dysfunction in septic mice and protects vascular endothelial cells from injury (8). Nod-like receptor protein 3 (NLRP3), which is a macromolecular complex protein and an inflammasome that can be activated by the hyperglycemia of islet cells and the deposition of amyloid polypeptides, plays an important role during the progression of diabetes $(9,10)$. Another study showed that NLRP3 in cardiac microvascular endothelial cells (CMECs) is activated when the body is in a state of myocardial ischemia reperfusion, which leads to myocardial cell injury (11).

According to the targeted prediction by TargetScan, miR-223-3p and NLRP3 have target binding sites. However, the role of miR-223-3p in the endothelial cell injury of diabetic patients has rarely been studied. Therefore, in order to confirm the conjecture that miR-223-3p can inhibit the injury of CMECs in diabetic patients by regulating the expression of NLRP3, a mouse model of diabetes was studied. 


\section{Materials and methods}

Materials and reagents. Twenty C57BL/6J female mice (purchased from the Experimental Animal Center of Sun Yat-sen University, Guangzhou, China), 4 weeks of age, with a body mass of $\sim 30 \mathrm{~g}$, were selected and randomized into the control and model groups ( $\mathrm{n}=10$ each). The mice were fed at $20-25^{\circ} \mathrm{C}$ with free access to food and water, with relative humidity of $40-75 \%$ and normal circadian rhythm. Mouse cardiac microvascular endothelial cells (MCMECs; cat. no. CP-M129) were purchased from Procell Life Science \& Technology Co., Ltd. and were frozen in liquid nitrogen. DMEM (high glucose) was purchased from Gibco (Thermo Fisher Scientific, Inc.). Fetal bovine serum (FBS) and trypsin were purchased from HyClone (GE Healthcare Life Sciences). TRIzol and reverse transcription kits were purchased from Takara Bio, Inc. RT-qPCR kit was purchased from Beijing Transgen Biotech Co., Ltd. qPCR fluorophore SYBR-Green (SY1020) was purchased from Beijing Solarbio Science \& Technology Co., Ltd. NLRP3 primary antibody (ab214185) was purchased from Abcam. Primary antibodies ( $\beta$-actin, Bax, caspase-3 and Bcl-2) and secondary antibody (goat anti-rabbit) were purchased from Shanghai Universal Biotech Co., Ltd. (cat. nos. 4970S, 2772S, 9662S, 3498S and A25012, respectively). Radioimmunoprecipitation assay buffer (SS0892) was purchased from Beijing Xinhua Lvyuan Technology Co., Ltd. ECL developing solution (GOYC3194) was purchased from Shanghai Guyan Industry Co., Ltd. Annexin V-FITC/PI apoptosis detection kit was purchased from Jiangsu KeyGEN Biotech Co., Ltd. Lipofectamine ${ }^{\mathrm{TM}}$ 2000 was purchased from Invitrogen (Thermo Fisher Scientific, Inc.). A dual luciferase reporter gene assay kit (D0010) was purchased from Beijing Solarbio Science \& Technology Co., Ltd. RT-qPCR primers were designed and synthesized by Shanghai GenePharma Co., Ltd. The study was approved by the Ethics Committee of The Third Affiliated Hospital of Nanchang University (Nanchang, China).

Modeling. Diabetic mice were modeled through high glucose and high fat (HGHF) diet. Sucrose, cream, premix, and water were mixed at 2:4:1:3 and heated to prepare a suspended HGHF emulsion. Streptozotocin powder injection (purchased from Sigma-Aldrich; Merck KGaA) was dissolved with $1 \%$ citrate buffer solution and degermed by a bacteria-proof filter, so as to prepare a streptozotocin solution. Before modeling, the mice in the three groups fasted overnight. Then, mice in the model and miR-223-3p groups were injected with streptozotocin solution $(35 \mathrm{mg} / \mathrm{kg})$ each time for 5 consecutive days, and fed with HGHF emulsion $(3 \mathrm{ml})$ each time for 6 consecutive weeks at the same time. At $48 \mathrm{~h}$ after the injection, the blood was drawn from the mouse tail vein for detecting blood glucose. The modeling was successful if the blood glucose was $>16.5 \mathrm{mmol} / \mathrm{l}$. After the blood glucose was elevated, the mice were injected through the tail vein with the drug delivery system $(1 \mathrm{mg} / \mathrm{kg})$ that was synthetized by neutral fat emulsion and miR-223-3p-mimics every 3 days, until the experiment was concluded. Mice in the control group were injected with normal saline (the same dose as that of streptozotocin solution) for 5 consecutive days. Six weeks later, the mice in both groups were sacrificed by cervical dislocation, to obtain the left ventricular tissues for detecting miR-223-3p and NLRP3 mRNA.

Cell modeling. MCMECs stored in liquid nitrogen were taken out and resuscitated in an incubator at $37^{\circ} \mathrm{C}$, then placed in a medium containing $10 \%$ FBS and cultured in an incubator at $37^{\circ} \mathrm{C}$ with $5 \% \mathrm{CO}_{2}$. After the adherent growth reached $80 \%$, the cells were washed with PBS, digested with $25 \%$ trypsin, and then cultured in the environment containing $10 \%$ culture fluid at $37^{\circ} \mathrm{C}$ with $5 \% \mathrm{CO}_{2}$ for passage. After the passage, cells in logarithmic growth phase were selected for grouping and transfection. They were divided into normal, model, blank carrier, miR-223-3p-mimics, and miR-223-3p-inhibitor groups. Cells in the normal and model groups were not transfected. However, the cells in the blank carrier group were transfected with miR-NC, the cells in the miR-223-3p-mimics group were transfected with miR-223-3p-mimics, and the cells in the miR-223-3p-inhibitor group were transfected with miR-223-3p-inhibitor. The specific steps were as follows: The cells were inoculated in a 6-well plate at $3 \times 10^{5}$ cells/well, and then Lipofectamine ${ }^{\mathrm{TM}} 2000$ was diluted and mixed with DNA according to the manufacturer's instructions of Lipofectamine 2000 transfection kit. The mixture was allowed to stand at room temperature for $5 \mathrm{~min}$, evenly mixed with cells, and then transfected for $48 \mathrm{~h}$ at $37^{\circ} \mathrm{C}$ with $5 \% \mathrm{CO}_{2}$. After transfection, the expression levels of miR-223-3p and NLRP3 in each group were detected, and cell models were established. Cells in the normal group continued to be cultured in the environment containing $10 \%$ culture fluid at $37^{\circ} \mathrm{C}$ with $5 \% \mathrm{CO}_{2}$. Cells in each group were placed in a high-glucose medium containing $10 \%$ FBS and glucose at $25 \mathrm{mmol} / \mathrm{l}$, added with palmitic acid at $300 \mu \mathrm{mol} / \mathrm{l}$ to simulate a high-fat environment, and then cultured in an incubator at $37^{\circ} \mathrm{C}$ with $5 \% \mathrm{CO}_{2}$. All the cells were continuously cultured for $24 \mathrm{~h}$, and subsequent experiments were carried out.

RT-qPCR detection of miR-223-3p and NLRP3 mRNA in tissues and cells. The left ventricular tissues of the mice in the two groups were ground and prepared into a suspension. TRIzol reagent was used to extract total RNA from miR-223-3p and NLRP3 mRNA in the suspension and cells. An ultraviolet spectrophotometer was used to detect its purity and concentration. Next, $5 \mu \mathrm{g}$ of total RNA were reversely transcribed into cDNA according to the manufacturer's instructions of the reverse transcription kit, and the parameters were $37^{\circ} \mathrm{C}$ for $15 \mathrm{~min}, 42^{\circ} \mathrm{C}$ for $42 \mathrm{~min}$, and $70^{\circ} \mathrm{C}$ for 5 min. The transcribed cDNA was used for PCR amplification, with $\beta$-actin as an internal reference for NLRP3 mRNA and U6 as an internal reference for miR-223-3p. qPCR was performed using the SYBR-Green fluorophore (Beijing Solarbio Science \& Technology Co., Ltd.). Primer sequences are shown in Table I. PCR conditions for miR-223-3p were as follows: Pre-denaturation at $95^{\circ} \mathrm{C}$ for $15 \mathrm{~min}$, then at $94^{\circ} \mathrm{C}$ for $15 \mathrm{sec}$ and at $55^{\circ} \mathrm{C}$ for $40 \mathrm{sec}$ for 40 cycles, finally extension at $70^{\circ} \mathrm{C}$ for $30 \mathrm{sec}$. PCR conditions for NLRP3 mRNA were as follows: Pre-denaturation at $95^{\circ} \mathrm{C}$ for $2 \mathrm{~min}$, then at $95^{\circ} \mathrm{C}$ for $10 \mathrm{sec}$ and at $60^{\circ} \mathrm{C}$ for $40 \mathrm{sec}$ for 40 cycles, final extension at $72^{\circ} \mathrm{C}$ for $90 \mathrm{sec}$. The $2^{-\mathrm{ACq}}$ method (12) was used to express the relative expression levels of genes, and a PCR instrument was used for fluorescence quantitative PCR. The experiment was carried out 3 times. 
Table I. Primer sequences.

Genes

Upstream primers

Downstream primers

$\operatorname{miR}-223-3 p$

U6

NLRP3 mRNA

$\beta$-actin

\author{
5'-GTGCAGGGTCCGAGGT-3' \\ 5'-CTCGCTTCGGCAGCACA-3' \\ 5'-GCAGCAAACTGGAAAGGAAG-3' \\ 5'-AGGGGCCGGACTCGTCATACT-3'
}

\author{
5'-CGGGCTGTCAGTTTGTCA-3' \\ 5'-AACGCTTCACGAATTTGCGT-3' \\ 5'-CTTCTCTGATGAGGCCCAAG-3' \\ 5'-GGCGGCACCACCATGTACCCT-3'
}

Western blot analysis of expression levels of NLRP3 and apoptosis-related proteins. The cells and tissues were homogenized in a radioimmunoprecipitation assay buffer. BCA protein assay kit was used to determine the concentration of proteins. Approximately $100 \mu \mathrm{g}$ of protein were loaded per lane, and 5\% concentrated SDS-PAGE gel and 15\% separating gel were used for electrophoresis. Then, the gels were transferred to a $0.45-\mu \mathrm{m}$ PVDF membrane that was sealed with $5 \%$ skimmed milk powder at room temperature for $2 \mathrm{~h}$. The cells were added with mouse monoclonal primary antibodies NLRP3 (1:500), Bax (1:500), caspase-3 (1:500), Bcl-2 (1:500), and $\beta$-actin $(1: 1,000)$, and then incubated overnight at $4{ }^{\circ} \mathrm{C}$. Next, the cells were added with HRP-labeled goat anti-rabbit antibody $(1: 1,000)$, incubated at $37^{\circ} \mathrm{C}$ for $1 \mathrm{~h}$, and rinsed with PBS solution. Finally, the cells were developed with ECL developing solution. The protein bands were scanned and their gray values were analyzed using Quantity One software (Bio-Rad Laboratories, Inc.). The relative expression level of the protein $=($ the gray value of the target protein band $) /($ the gray value of $\beta$-actin protein band).

Flow cytometry detection of apoptosis of MCMECs. Annexin V-FITC/PI double staining combined with flow cytometry was used to detect apoptosis. After cell modeling, MCMECs in each group were inoculated in a 6 -well plate at $3 \times 10^{5}$ cells/well and then incubated for $24 \mathrm{~h}$. Then, they were rinsed twice with PBS and added with Annexin V-FITC (5 $\mu \mathrm{l})$. After 10-min reaction at room temperature, the cells were added with PI $(10 \mu \mathrm{l})$ and incubated at room temperature in the dark for $20 \mathrm{~min}$. Finally, the flow cytometer was used to detect apoptosis. The experiment was carried out 3 times. FlowJo v.10 software (FlowJo LLC) was used for analysis.

Dual-luciferase reporter gene assay. The downstream target genes of miR-223-3p were predicted using TargetScan 7.1 (http://www.targetscan.org/vert_71/). Oligonucleotides containing NLRP3 target sequence were amplified and cloned into wild type (WT) pmirGLO plasmids (Biovector Co., Ltd; Biovector105805). pmirGLO-NLRP3 3' UTR-WT and pmirGLO-NLRP3 3' UTR-mutant (Mut) were constructed and then transferred to the downstream of the luciferase reporter genes, so as to sequence and identify the constructed plasmids. NLRP3 3' UTR-WT, NLRP3 3' UTR-Mut, miR-223-3p-mimics, miR-223-3p-inhibitor, and miR-NC were transferred into the MCMECs using Lipofectamine 2000 kit. At $48 \mathrm{~h}$ after the transfection, a dual-luciferase reporter gene assay kit (Promega Corp.) was used to determine luciferase activity, with Renilla luciferase activity considered as the standard.
Statistical analysis. SPSS 18.0 [Bizinsight (Beijing) Information Technology Co., Ltd.] was used to statistically analyze the data. GraphPad Prism 6 (GraphPad Software, Inc.) was used to plot the figures. Measurement data were expressed as the mean \pm standard deviation, and independent samples t-test was used for comparisons between two groups, whereas analysis of variance with LSD post hoc test were used for comparisons between multiple groups. $\mathrm{P}<0.05$ was considered to indicate a statistically significant difference.

\section{Results}

Target relationship between miR-223-3p and NLRP3. According to the prediction by TargetScan, bases 406-412 of NLRP3 3' UTR were binding sites of miR-223-3p. Dual-luciferase reporter gene assay was conducted to confirm the direct interaction between miR-223-3p and NLRP3. Co-transfection with miR-223-3p reduced the luciferase activity of plasmids containing fragments of NLRP3 3' UTR-WT (Fig. 1A). These results indicated that miR-223-3p directly interacted with NLRP3 3' UTR. The results of RT-qPCR after transfection revealed that the expression of miR-223-3p in the miR-223-3p-mimics group was significantly higher than that in the miR-NC group, while the expression in the miR-223-3p-inhibitor group was significantly lower than that in the miR-NC group (Fig. 1B). The results of western blot analysis revealed that the expression of NLRP3 significantly decreased in the MCMECs transfected with miR-223-3p-mimics, while NLRP3 expression significantly increased in the MCMECs transfected with miR-223-3p-inhibitor $(\mathrm{P}<0.05)$ (Fig. 1C).

Expression levels of miR-223-3p,NLRP3, and apoptosis-related proteins in heart tissue. The mice in the miR-223-3p-mimics group were injected through the tail vein with the drug delivery system that was synthetized by neutral fat emulsion and miR-223-3p-mimics. The results revealed that compared with those in the model group, the mice in the control and miR-223-3p-mimics groups had significantly higher expression of miR-223-3p. However, significantly lower mRNA and protein expression levels of NLRP3 were observed. Additionally, they had significantly lower protein expression levels of Bax and caspase-3; however,a significantly higher protein expression of Bcl-2 was observed ( $\mathrm{P}<0.05)$ (Fig. 2).

Effects of miR-233-3p on NLRP3 expression and endothelial cell apoptosis. After cell modeling and culture in the HGHF environment, the cells in the model and blank carrier groups had significantly lower expression of miR-223-3p, compared 
A

\begin{tabular}{|c|c|c|c|c|}
\hline & and mIRNA & typo & score & percentllo \\
\hline Position 406-412 of NLRP3 3 ' UTR & 5' ...CGCUAUCUUUCUAUUAACUGACC... & $\underset{\mathrm{mB}}{7 \mathrm{mer}-}$ & -0.30 & 94 \\
\hline
\end{tabular}

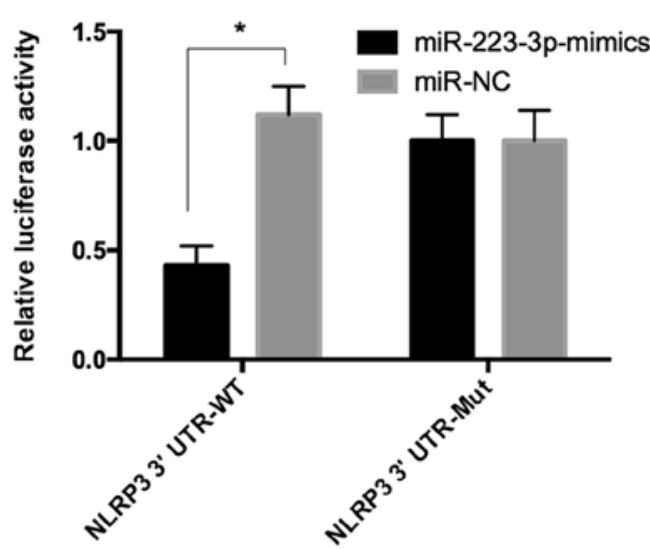

C

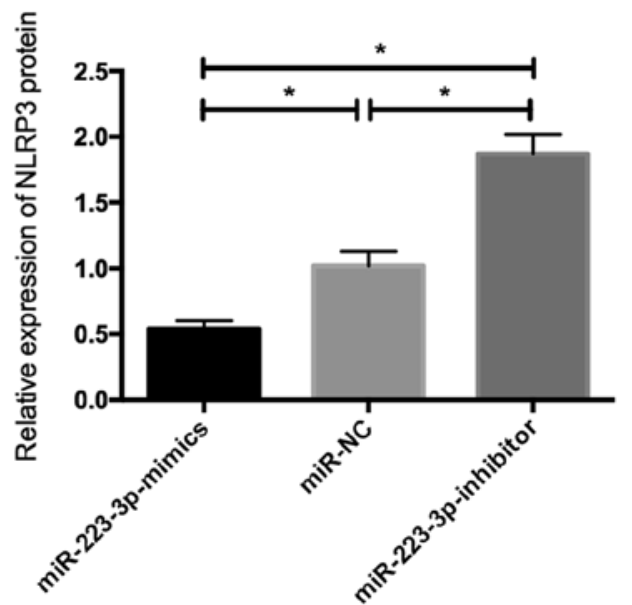

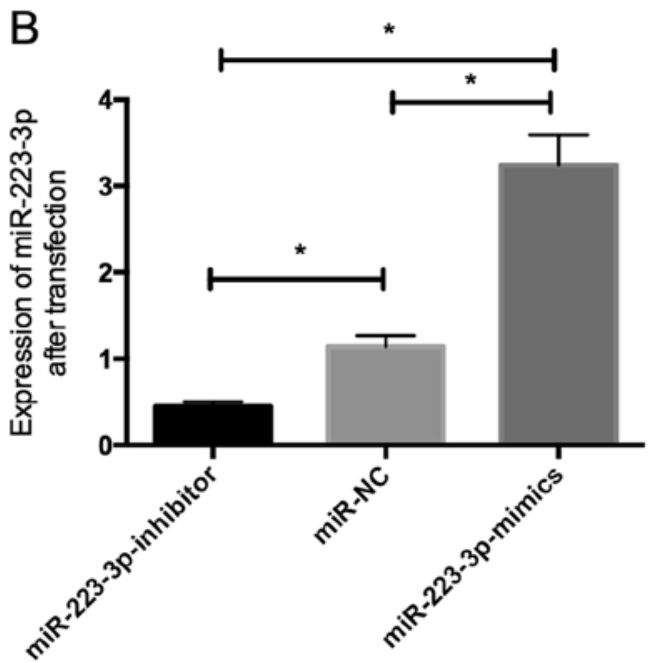

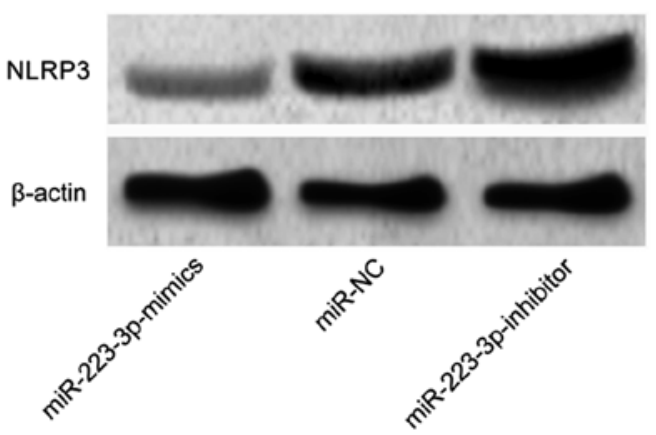

Figure 1. Target relationship between miR-223-3p and NLRP3. (A) There were binding sites between miR-223-3p and NLRP3. (B) Expression of miR-223-3p in MCMECs after transfection. (C) Effects of miR-223-3p on the protein expression of NLRP3. *P<0.05. NLRP3, Nod-like receptor protein 3; MCMECs, mouse cardiac microvascular endothelial cells.
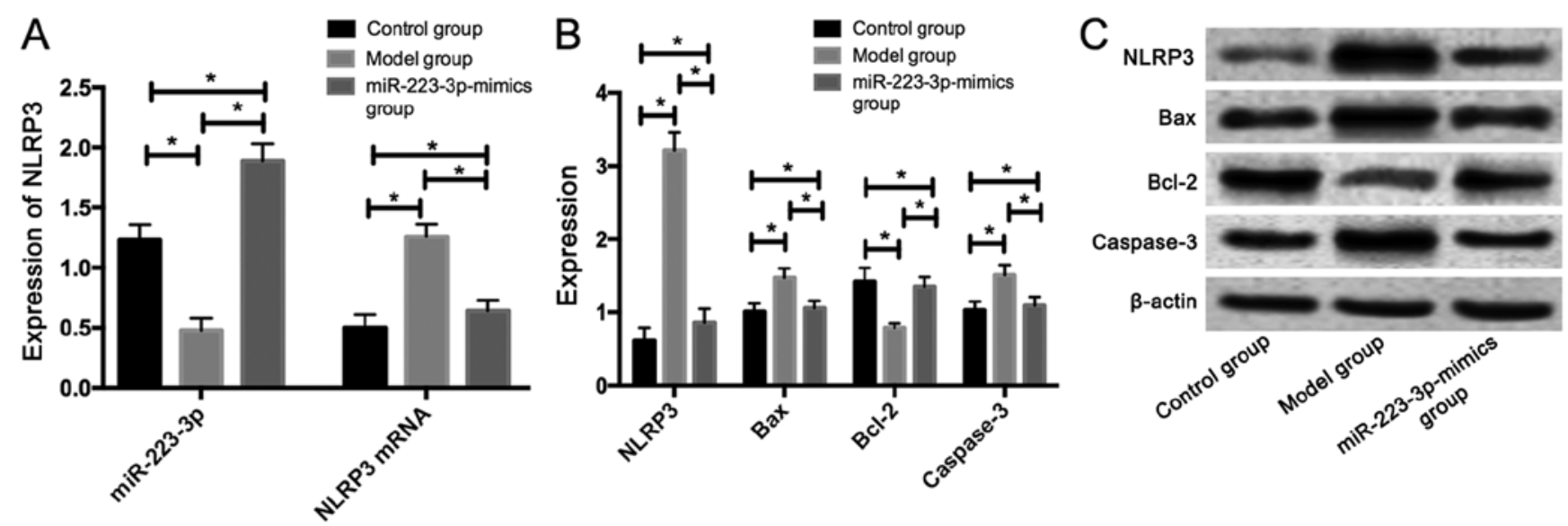

Figure 2. Expression levels of miR-223-3p, NLRP3, and apoptosis-related proteins in heart tissue. (A) Expression levels of miR-223-3p and NLRP3 mRNA in heart tissue. (B) Expression levels of NLRP3 and apoptosis-related proteins in heart tissue. (C) Western blots. *P<0.05. NLRP3, Nod-like receptor protein 3.

with those in the normal group; however, significantly higher mRNA and protein expression levels of NLRP3 $(\mathrm{P}<0.05)$ were observed. Additionally, compared with those in the model and blank carrier groups, the cells in the
miR-223-3p-mimics group had significantly higher expression of miR-223-3p, but significantly lower mRNA and protein expression levels of NLRP3 $(\mathrm{P}<0.05)$. Also, the cells in the miR-223-3p-inhibitor group had significantly higher mRNA 

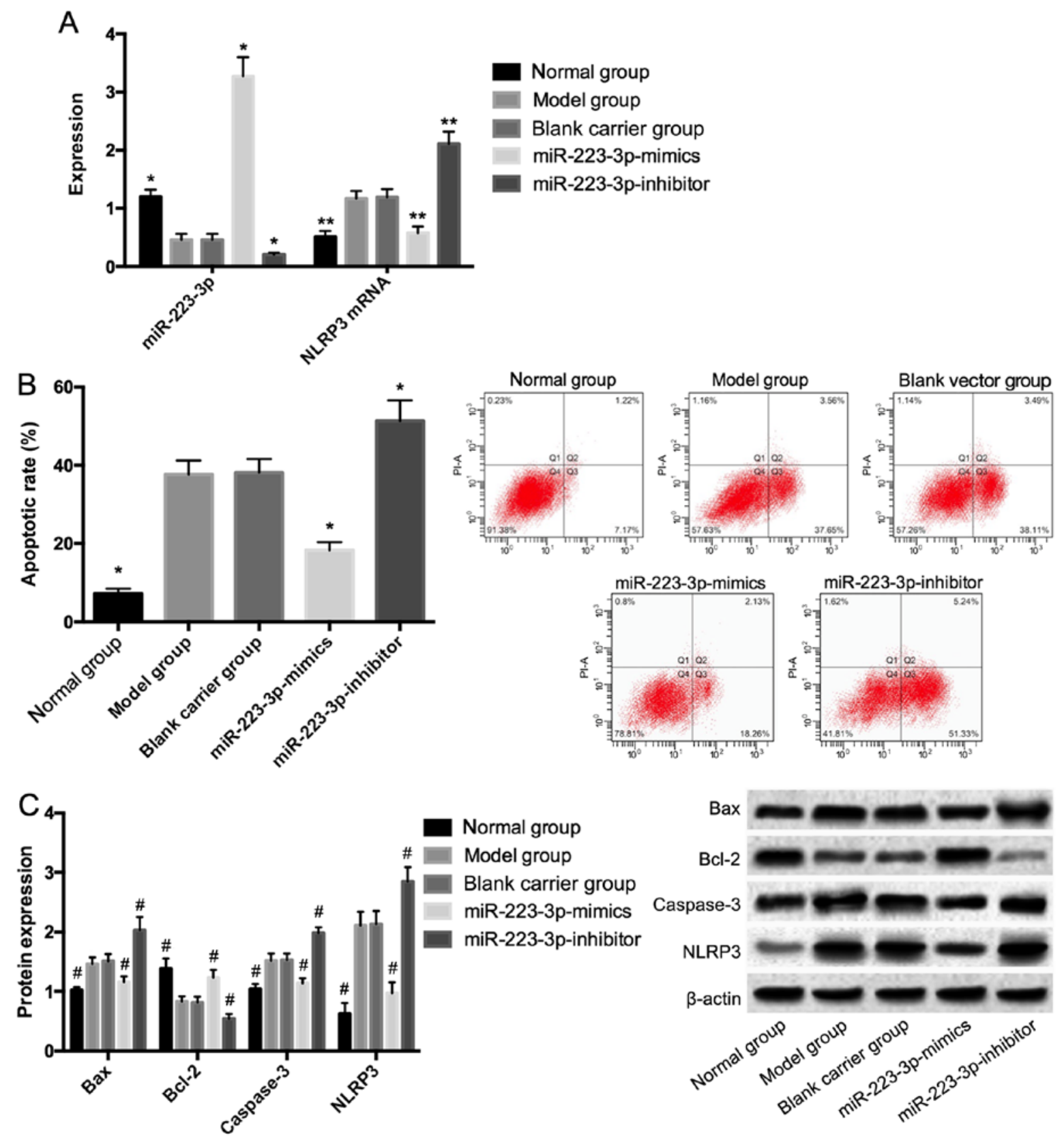

Figure 3. Effects of miR-233-3p on NLRP3 expression and endothelial cell apoptosis. (A) Expression levels of miR-223-3p and NLRP3 mRNA. (B) Apoptotic rates. (C) Expression levels of apoptosis-related proteins. ${ }^{*} \mathrm{P}<0.05,{ }^{* *} \mathrm{P}<0.05$, compared with the model and blank carrier groups. ${ }^{*} \mathrm{P}<0.05$, compared with the model and blank carrier groups for the same protein. NLRP3, Nod-like receptor protein 3.

and protein expression levels of NLRP3 $(\mathrm{P}<0.05)$, compared with those in the model and blank carrier groups. Compared with those in the normal group, the cells in the model and blank carrier groups had a significantly higher apoptotic rate, significantly higher protein expression levels of Bax and caspase -3 , but significantly lower protein expression of $\mathrm{Bcl}-2(\mathrm{P}<0.05)$. Compared with those in the model and blank carrier groups, the cells in miR-223-3p-mimics group had a significantly lower apoptotic rate, and significantly lower protein expression levels of Bax and caspase-3. However, a significantly higher protein expression of Bcl-2 was observed $(\mathrm{P}<0.05)$. In addition, compared with those in the model and blank carrier groups, the cells in the miR-223-3p-inhibitor group had a significantly higher apoptotic rate, significantly higher protein expression levels of Bax and caspase-3; whereas a significantly lower protein expression of $\mathrm{Bcl}-2$ was observed $(\mathrm{P}<0.05)$ (Fig. 3).

\section{Discussion}

Diabetes is a metabolic disease caused by multiple factors which is clinicopathologically characterized by hyperglycemia (13). Diabetes leads to metabolic disorders and a series of complications, which not only seriously affect the patients' quality of life, but also bring heavy burdens to the family of the patients and society $(14,15)$. Diabetic cardiomyopathy is a common complication of diabetes and is mainly caused by the dysfunction of CMECs (16). 
NLRP3 is currently the most widely studied inflammasome. According to previous studies, during the pathogenesis of diabetes, the increase of inflammatory responses over-activates NLRP3 and the over-activation induces the production of a large amount of IL-1 $\beta$, which further causes damage to islet cells and results in insulin resistance. This shows that NLRP3 is closely related to diabetes $(17,18)$. A previous study has shown that diabetic cardiovascular diseases have the characteristics of inflammatory diseases, and the over-activation of NLRP3 may also lead to vascular dysfunction in diabetic patients (19). The results of the targeted prediction by TargetScan revealed that miR-223-3p and NLRP3 have target binding sites. Another study has reported that miR-223-3p is a new biomarker and plays a protective role in cardiovascular and related fields (20). Therefore, it is speculated that miR-223-3p can reduce the diabetes-induced injury of CMECs through regulating NLRP3 in a targeted manner.

In the present study, mice were modeled for diabetes through HGHF diet, and miR-223-3p and NLRP3 expression levels in the heart tissue of diabetic and normal mice were detected. The results revealed that compared with those in the model group, the mice in the control group had significantly higher expression of miR-223-3p, and significantly lower mRNA and protein expression levels of NLRP3, suggesting that NLRP3 may cause diabetic vascular injury. Mice in the miR-223-3p-mimics group were injected through the tail vein with the drug delivery system that was synthetized by neutral fat emulsion and miR-223-3p-mimics. The results revealed that compared with those in the model group, mice in the miR-223-3p-mimics group had significantly higher expression of miR-223-3p; however, significantly lower mRNA and protein expression levels of NLRP3 were observed. Additionally, they had significantly lower protein expression levels of Bax and caspase-3; whereas a significantly higher protein expression of Bcl-2 was observed. These findings reveal that miR-223-3p may regulate NLRP3 and apoptosis-related proteins. A previous study found a significant increase in the serum NLRP3 of patients with diabetic cardiomyopathy (21), which is consistent with our conclusions.

In a study of miR-223-3p on patients with rheumatic heart disease, it was shown that miR-223-3p inhibits the activation of $\mathrm{T}$ cells and reduces myocardial damage and myocardial cell apoptosis by regulating inflammatory cytokines that are secreted by inflammatory cells (22). This indicates that miR-223-3p can regulate inflammatory cytokines and thereby protect angiocarpy. As known, glycolipid metabolic abnormalities are of great significance for the development and progression of diabetes $(23,24)$, but whether NLRP3 could be regulated by miR-223-3p has rarely been explored. Moreover, in vivo experiments alone cannot prove that miR-223-3p can reduce the injury of CMECs and regulate NLRP3. Therefore, an endothelial cell injury model was established in vitro through HGHF diet. The results revealed that after cell modeling and culture in the HGHF environment, the cells in the miR-223-3p overexpression group had significantly higher expression of miR-223-3p, compared with those in the model group, while cells in the low miR-223-3p expression group had significantly lower expression of miR-223-3p. Compared with those in the model and blank carrier groups, the cells in the miR-223-3p overexpression group had significantly lower mRNA expression of NLRP3, while cells in the low miR-223-3p expression group had significantly higher mRNA expression of NLRP3. Apoptosis in each group was detected and compared. The results revealed that after modeling, the protein expression levels of Bax and NLRP3 in the normal group were significantly lower than those in the model, blank carrier, and miR-223-3p overexpression groups, while the protein expression levels of Bax, caspase-3, and NLRP3 in the miR-223-3p overexpression group were significantly lower than those in the model and blank carrier groups. The protein expression of Bcl-2 in the normal group was significantly higher than that in the model, blank carrier, and miR-223-3p overexpression groups, while the protein expression in the miR-223-3p overexpression group was significantly higher than that in the model and blank carrier groups. These findings suggest that the overexpression of miR-223-3p in injured endothelial cells can inhibit the expression of NLRP3, thus protecting endothelial cells and reducing apoptosis of CMECs. In addition, NLRP3 may inhibit endothelial cell apoptosis by affecting apoptosis-related proteins Bax and caspase-3, as well as anti-apoptotic protein $\mathrm{Bcl}-2$. A previous study has shown that long-term hyperglycemia leads to massive generation of oxygen-free radicals, which causes damage to DNA in cells and results in cell damage with apoptosis as the main manifestation (25). Although NLRP3 is a target gene of miR-223-3p, the regulatory mechanism of miR-223-3p on NLRP3 remains unclear, which is also a deficiency of this study.

In conclusion, miR-223-3p can inhibit the diabetes-induced apoptosis of CMECs by regulating the expression of NLRP3, and protect CMECs from injury. However, the regulatory mechanism of miR-223-3p on NLRP3 and the regulatory mechanism of NLRP3 on apoptosis-related proteins were not explored. These aspects need further study to provide more theoretical data for the treatment and prevention of diabetic patients with cardiovascular injury.

\section{Acknowledgements}

Not applicable.

\section{Funding}

No funding was received.

\section{Availability of data and materials}

The datasets used and/or analyzed during the present study are available from the corresponding author on reasonable request.

\section{Authors' contributions}

$\mathrm{BD}$ and $\mathrm{YiH}$ conceived and designed the study. BD, XS, HZ and $\mathrm{YaH}$ were responsible for the collection and analysis of the experimental data. YiH and XS interpreted the data and drafted the manuscript. BD and YaH revised the manuscript critically for important intellectual content. All authors read and approved the final manuscript. 


\section{Ethics approval and consent to participate}

The study was approved by the Ethics Committee of The Third Affiliated Hospital of Nanchang University (Nanchang, China).

\section{Patient consent for publication}

Not applicable.

\section{Competing interests}

The authors declare that they have no competing interests.

\section{References}

1. Chamberlain JJ, Rhinehart AS, Shaefer CF Jr and Neuman A: Diagnosis and management of diabetes: Synopsis of the 2016 American Diabetes Association Standards of Medical Care in Diabetes. Ann Intern Med 164: 542-552, 2016.

2. Micha R, Peñalvo JL, Cudhea F, Imamura F, Rehm CD and Mozaffarian D: Association between dietary factors and mortality from heart disease, stroke, and type 2 diabetes in the United States. JAMA 317: 912-924, 2017.

3. Zhao W, Rasheed A, Tikkanen E, Lee JJ, Butterworth AS, Howson JMM, Assimes TL, Chowdhury R, Orho-Melander M, Damrauer S, et al; CHD Exome+ Consortium; EPIC-CVD Consortium; EPIC-Interact Consortium; Michigan Biobank: Identification of new susceptibility loci for type 2 diabetes and shared etiological pathways with coronary heart disease. Nat Genet 49: 1450-1457, 2017.

4. Parenti A, Pala L, Paccosi S and Rotella CM: Potential role for dendritic cells in endothelial dysfunction, diabetes and cardiovascular disease. Curr Pharm Des 23: 1435-1444, 2017.

5. Rawal S, Munasinghe PE, Shindikar A, Paulin J, Cameron V, Manning P, Williams MJ, Jones GT, Bunton R, Galvin I, et al: Down-regulation of proangiogenic microRNA-126 and microRNA-132 are early modulators of diabetic cardiac microangiopathy. Cardiovasc Res 113: 90-101, 2017.

6. Dai GH, Ma PZ, Song XB, Liu N, Zhang T and Wu B: MicroRNA-223-3p inhibits the angiogenesis of ischemic cardiac microvascular endothelial cells via affecting RPS6KB1/hif-1a signal pathway. PLoS One 9: e108468, 2014.

7. Liu X, Zhang Y, Du W, Liang H, He H, Zhang L, Pan Z, Li X, $\mathrm{Xu} \mathrm{C}$, Zhou Y, et al: MiR-223-3p as a novel microRNA regulator of expression of voltage-gated $\mathrm{K}^{+}$channel $\mathrm{Kv} 4.2$ in acute myocardial infarction. Cell Physiol Biochem 39: 102-114, 2016.

8. Wang X, Huang W, Yang Y, Wang Y, Peng T, Chang J, Caldwell CC, Zingarelli B and Fan GC: Loss of duplex miR-223 (5p and 3p) aggravates myocardial depression and mortality in polymicrobial sepsis. Biochim Biophys Acta 1842: 701-711, 2014.

9. Yi H, Peng R, Zhang LY, Sun Y, Peng HM, Liu HD, Yu LJ, Li AL, Zhang YJ, Jiang WH, et al: LincRNA-Gm4419 knockdown ameliorates NF- $\mathrm{KB} / \mathrm{NLRP} 3$ inflammasome-mediated inflammation in diabetic nephropathy. Cell Death Dis 8: e2583, 2017.

10. Stienstra R, van Diepen JA, Tack CJ,Zaki MH, van de Veerdonk FL, Perera D, Neale GA, Hooiveld GJ, Hijmans A, Vroegrijk I, et al: Inflammasome is a central player in the induction of obesity and insulin resistance. Proc Natl Acad Sci USA 108: 15324-15329, 2011.

11. Wang L, Chen Y, Li X, Zhang Y, Gulbins E and Zhang Y: Enhancement of endothelial permeability by free fatty acid through lysosomal cathepsin B-mediated Nlrp3 inflammasome activation. Oncotarget 7: 73229-73241, 2016.
12. Zhou W, Wang G, Zhao X, Xiong F, Zhou S, Peng J, Cheng Y, $\mathrm{Xu} \mathrm{S}$ and $\mathrm{Xu} \mathrm{X}$ : A multiplex qPCR gene dosage assay for rapid genotyping and large-scale population screening for deletional $\alpha$-thalassemia. J Mol Diagn 5: 642-651, 2013.

13. Soliman EZ, Backlund JC, Bebu I, Orchard TJ, Zinman B and Lachin JM; DCCT/EDIC Research Group: Electrocardiographic abnormalities and cardiovascular disease risk in type 1 diabetes: The Epidemiology of Diabetes Interventions and Complications (EDIC) Study. Diabetes Care 40: 793-799, 2017.

14. Trikkalinou A, Papazafiropoulou AK and Melidonis A: Type 2 diabetes and quality of life. World J Diabetes 8: 120-129, 2017.

15. Cai H, Li G, Zhang P, Xu D and Chen L: Effect of exercise on the quality of life in type 2 diabetes mellitus: A systematic review. Qual Life Res 26: 515-530, 2017.

16. Guo R and Nair S: Role of microRNA in diabetic cardiomyopathy: From mechanism to intervention. Biochim Biophys Acta Mol Basis Dis 1863: 2070-2077, 2017.

17. Ye Y, Bajaj M, Yang HC, Perez-Polo JR and Birnbaum Y: SGLT-2 inhibition with dapagliflozin reduces the activation of the Nlrp3/ASC inflammasome and attenuates the development of diabetic cardiomyopathy in mice with type 2 diabetes. Further augmentation of the effects with saxagliptin, a DPP4 inhibitor. Cardiovasc Drugs Ther 31: 119-132, 2017.

18. Yang X, Lu F, Li L, Li J, Luo J, Zhang S, Liu X and Chen G: Wu-Mei-wan protects pancreatic $\beta$ cells by inhibiting NLRP3 inflammasome activation in diabetic mice. BMC Complement Altern Med 19: 35, 2019.

19. Liu Y, Lian K, Zhang L, Wang R, Yi F, Gao C, Xin C, Zhu D, Li Y, Yan W, et al: TXNIP mediates NLRP3 inflammasome activation in cardiac microvascular endothelial cells as a novel mechanism in myocardial ischemia/reperfusion injury. Basic Res Cardiol 109: 415, 2014.

20. Chu M, Wu R, Qin S, Hua W, Shan Z, Rong X, Zeng J, Hong L, Sun Y, Liu Y, et al: Bone marrow-derived microRNA-223 works as an endocrine genetic signal in vascular endothelial cells and participates in vascular injury from Kawasaki disease. J Am Heart Assoc 6: e004878, 2017.

21. Zhang J, Xia L, Zhang F, Zhu D, Xin C, Wang H, Zhang F, Guo X, Lee Y, Zhang L, et al: A novel mechanism of diabetic vascular endothelial dysfunction: Hypoadiponectinemia-induced NLRP3 inflammasome activation. Biochim Biophys Acta Mol Basis Dis 1863: 1556-1567, 2017.

22. Castro-Villegas C, Pérez-Sánchez C, Escudero A, Filipescu I, Verdu M, Ruiz-Limón P, Aguirre MA, Jiménez-Gomez Y, Font P, Rodriguez-Ariza A, et al: Circulating miRNAs as biomarkers of therapy effectiveness in rheumatoid arthritis patients treated with anti-TNFo. Arthritis Res Ther 17: 49, 2015.

23. Akushevich I, Yashkin AP, Kravchenko J, Fang F, Arbeev K, Sloan F and Yashin AI: Identifying the causes of the changes in the prevalence patterns of diabetes in older U.S. adults: A new trend partitioning approach. J Diabetes Complications 32: 362-367, 2018.

24. Patterson R, McNamara E, Tainio M, de Sá TH, Smith AD, Sharp SJ, Edwards P, Woodcock J, Brage S and Wijndaele K: Sedentary behaviour and risk of all-cause, cardiovascular and cancer mortality, and incident type 2 diabetes: A systematic review and dose response meta-analysis. Eur J Epidemiol 33: 811-829, 2018

25. Kowluru RA, Kowluru V, Xiong Y and Ho YS: Overexpression of mitochondrial superoxide dismutase in mice protects the retina from diabetes-induced oxidative stress. Free Radic Biol Med 41: 1191-1196, 2006.

(i) $($ ) This work is licensed under a Creative Commons Attribution-NonCommercial-NoDerivatives 4.0 International (CC BY-NC-ND 4.0) License. 